\title{
Family satisfaction with critical care in the united kingdom
}

\author{
SE Wright ${ }^{1 *}$, SE Harvey ${ }^{2}$, E Walmsley ${ }^{2}$, P Ferrando-Vivas ${ }^{2}$, DA Harrison², KM Rowan² \\ From ESICM LIVES 2015 \\ Berlin, Germany. 3-7 October 2015
}

\section{Introduction}

A number of tools have been developed to seek the views of family members of critically ill patients but the most widely validated is the Family Satisfaction in the Intensive Care Unit 24-item questionnaire (FS-ICU24), [1] which assesses overall family satisfaction, satisfaction with care and satisfaction with decision-making.

\section{Objectives}

To assess family satisfaction with critical care in the United Kingdom using the FS-ICU-24, compare results internationally, and explore the impact of family, patient and other factors on comparisons between ICUs.

\section{Methods}

The Family Reported Experiences Evaluation (FREE) Study recruited family members of patients staying at least 24 hours in 20 participating adult general ICUs between May 2013 and June 2014. Consenting family members were sent a postal questionnaire three weeks after the patient died or was discharged from ICU. Up to four family members were recruited per patient. Multilevel multivariable models were used to identify factors associated with satisfaction.

\section{Results}

12,346 family members of 6380 patients were recruited to the FREE Study and 7173 (58\%) family members of 4615 patients returned a completed questionnaire. Multiple imputation of missing item response enabled inclusion of all responders. Overall, satisfaction scores were high (mean overall family satisfaction 80 , satisfaction with care 83 , satisfaction with decision-making 75 out of 100) and were similar to other reports, internationally. Satisfaction was higher for family members of ICU non-survivors.

${ }^{1}$ Freeman Hospital, Newcastle upon Tyne, United Kingdom

Full list of author information is available at the end of the article
Factors associated with overall satisfaction for family members of ICU survivors were family member age, ethnicity, relationship (to patient), visit frequency and patient acute severity of illness and receipt of invasive mechanical ventilation. Factors associated with overall satisfaction for family members of ICU non-survivors were patient age, acute severity of illness and duration of ICU stay. No other factors (of those explored) were associated. Significant variation existed across ICUs which reduced following adjustment for family and patient factors, resulting in fewer ICUs being identified as potential outliers.

\section{Conclusions}

The large sample size and robust multilevel multivariable modelling of factors associated with overall satisfaction indicated the need for adjustment for these when comparing ICUs. The FREE Study and the FREE Study database are an important foundation and resource for future studies evaluating family satisfaction with critical care in the United Kingdom.

\section{Grant Acknowledgment}

NIHR Health Services and Delivery Research Programme.

Authors' details

${ }^{1}$ Freeman Hospital, Newcastle upon Tyne, United Kingdom. ${ }^{2}$ Intensive Care National Audit \& Research Centre, Clinical Trials Unit, London, United Kingdom.

Published: 1 October 2015

\section{Reference}

1. Wall RJ, Engelberg RA, Downey L, Heyland DK, Curtis JR: Refinement, scoring, and validation of the Family Satisfaction in the Intensive Care Unit (FS-ICU) survey. Crit Care Med 2007, 35:271-9.

doi:10.1186/2197-425X-3-S1-A22

Cite this article as: Wright et al:: Family satisfaction with critical care in the united kingdom. Intensive Care Medicine Experimental 2015 3(Suppl 1): A22. (c) 2015 Wright et al.; This is an Open Access article distributed under the terms of the Creative Commons Attribution License (http:// creativecommons.org/licenses/by/4.0), which permits unrestricted use, distribution, and reproduction in any medium, provided the original work is properly cited. 